\title{
Timely Prediction of Peak Seasonal Influenza Activity Estimation Using Sentinel Surveillance Data
}

\author{
Núria Torner ${ }^{1,2,3, *}$, Núria Soldevila ${ }^{3}$, Ana Martínez ${ }^{1}$, Tomas Pumarola ${ }^{4}$, Angela Dominguez $^{2,3}$ \\ ${ }^{1}$ Department of Health.Generalitat of Catalonia, Barcelona, Spain \\ ${ }^{2}$ Department of Public Health, University of Barcelona, Spain \\ ${ }^{3}$ CIBER Epidemiología y Salud Pública (CIBERESP), Spain \\ ${ }^{4}$ Service of Microbiology, H Clinic of Barcelona, Spain
}

\begin{abstract}
Influenza sentinel surveillance provides valuable information to help plan resources and preparedness. Study aim was to determine which age group could give timely prediction on onset of seasonal epidemic activity. Methods: Study period comprised influenza epidemic seasons 2006-2009. Incidence rates (IR) of influenza like illness (ILI), for 2 and 3 weeks prior to onset of epidemic activity by age group and peak epidemic activity weeks for general population were calculated. Rate Ratios (RR) between influenza virus positive samples (IV+) and ILI, between 2 and 3 weeks prior to epidemic ILI activity by age group and for peak activity period (PA) were obtained. Differences in rate proportions and their $95 \%$ confidence intervals were assessed by statistic $z$. Results: The highest RR between IV+ and ILI rates corresponded to $0-4 y$ (0.34; $95 \%$ CI: $0.28-0.41)$, and lowest $(0.26$; $95 \% \mathrm{CI}: 0.23-0.30)$ to $15-64 \mathrm{y}$. Considering two and three weeks prior to epidemic activity onset related to overall population IRs during PA period, the only RR above 1 corresponded to $0-4 y$ (1.38; $95 \%$ CI: $1.08,1.75)$ for two weeks and $(1.29 ; 95 \%$ CI:1.04,1.6) for three weeks. Conclusion: High correspondence between ILI and IV+ was observed in 0-4y prior to other age groups; in this age group rates of ILI during two and three consecutive weeks prior to increase above threshold activity can be used to predict the onset of peak epidemic activity in a timely manner.
\end{abstract}

Keywords Influenza, Incidence Rate, Surveillance

\section{Introduction}

Seasonal influenza epidemics are major public health concern causing tens of millions of respiratory disease and hundreds of thousands deaths worldwide. In addition emergence of new influenza strain demonstrating human-to-human transmission and against which there is no previous immunity resulting in a pandemic, stresses out the importance of early detection of disease activity. When this early detection can be followed by a rapid response, impact of both seasonal and pandemic influenza can be reduced. Influenza in children in well recognized as a yearly burden, with severe symptoms and complications responsible for hospitalizations in young children and at risk groups. Clinical diagnosis, similar to adults in children over $4 \mathrm{y}$, is much less feasible in young infants due to overlap of symptoms with other respiratory or gastrointestinal viral epidemics[1]. Confirmation of clinical diagnosis by means of laboratory techniques is of utmost importance especially in children because of the more atypical presentation features in this age group in comparison to adult population[2] and because the

* Corresponding author:

Nuria.torner@gencat.cat (Nuria Torner)

Published online at http://journal.sapub.org/xxx

Copyright (C) 2012 Scientific \& Academic Publishing. All Rights Reserved greater overall attack rate in children even in mild or moderate influenza activity seasons[3].

Influenza like illness (ILI) surveillance in Catalonia, with more than 20 years of experience in influenza sentinel surveillance, provides clinical and viral information on influenza activity and enables identification of circulating strains.

The emergence of a novel strain of influenza virus A (H1N1) in April 2009 focused attention on influenza surveillance capabilities worldwide. In consultations before the 2009 outbreak of influenza subtype H1N1, the World Health Organization had concluded that the world was unprepared to respond to an influenza pandemic, due in part to inadequate global surveillance and response capacity. Sentinel surveillance systems could enhance the quality of influenza epidemiologic and laboratory data and strengthen a country's capacity for seasonal, novel, and pandemic influenza detection and prevention[4].

Vaccine for influenza is known to have different efficacy and effectiveness as to reduction of transmission for different age groups[5,6]. Several studies on influenza mortality among elderly during time periods when schoolchildren were and were not vaccinated suggests that the infected children pose a risk to others including the elderly[7,8].

This study aimed to determine which age group can give a more timely prediction of the onset of peak epidemic activity during the first weeks prior to the onset of seasonal influenza 
and allow for adequate healthcare facility preparedness to cope with high disease burden as well as setting priority for vaccination policies by targeting not only age groups that are most vulnerable, but also those with the highest infection rate.

\section{Methods}

The Influenza surveillance network of Catalonia (PIDIRAC) compiles clinical and virological surveillance during seasonal influenza activity. Study period was taken from week 45 to 15 of three epidemic influenza surveillance seasons (2006-07, 2007-08 and 2008-09). ILI is defined as acute respiratory tract infection presenting with sudden onset of symptoms AND at least one of the following four systemic symptoms: fever or feverishness, malaise, headache, myalgia; AND at least one of the following three respiratory symptoms: cough, sore throat, and shortness of breath[9]. Threshold incidence rates for epidemic influenza activity are defined for each influenza season, using the mean incidence rates from preceding seasons. We defined as "peak epidemic activity" period the interval of weeks with an incidence rate for influenza like illness exceeding the threshold for that influenza season. Data was compiled through weekly influenza virus identification in nasal-pharynx and throat systematic sampling ( 2 first ILI consultations per week per physician), and incidence of ILI consultation reporting by the sentinel surveillance network (27 general practitioners and 29 pediatricians). Each sentinel physician has assigned a number of patients distributed into four age groups $0-4,5-14$, 15-64 and older than 64 years, covering about $1 \%$ of the population throughout Catalonia. Population surveyed each week, calculated according to sentinel physicians' weekly reporting activity, was used in the calculation of ILI IR. Virology assessment was carried out on swabs placed into modified Hanks transport media at the Microbiology Department of Hospital Clínic of Barcelona. Viral identification included Influenza virus A (IVA), B (IVB) and C (IVC) as well as other respiratory tract viruses (adenovirus, respiratory syncytial virus, rhinovirus, human parainfluenza 1,2,3,4 viruses, human coronavirus, enterovirus and human metapneumovirus). Techniques used were indirect immunofluorescent monoclonal antibody antigen detection (IFI), virus isolation in cell culture and isolate identification by means of inverse transcriptase polymerase chain reaction (RT-PCR).

Incidence rates of influenza like illness (ILI), positive influenza virus (IV+) and ILI for two and three weeks prior of onset epidemic activity by age group as well as peak epidemic activity weeks for general population were calculated. Rate Ratios (RR) between IV+ and ILI and between 2 and 3 weeks prior to epidemic ILI activity (2\&3) for each age group and for PA were obtained. The $95 \%$ confidence intervals (CI) were calculated assuming a Poisson distribution. Differences in rate proportions and their $95 \%$ confidence intervals were assessed by statistic $z$ and Mantel Hanzel's Woolf test. The level of statistical significance was established at $\alpha=0.05$.

\section{Results}

During the three epidemic seasons included in the study, a total of 2085 samples were collected from the sentinel influenza surveillance network clinicians. The age distribution of these samples were $30.6 \%$ from age group $0-4$ y; $25,0 \%$ from the 5-14 y. group; $40,0 \%$ from the $15-64$ y group and $4,5 \%$ from the $>64$ y group. The overall positivity rate for influenza virus ( $A$ and $B$ ) was significantly higher in the 5-14 $\mathrm{y}$ than in the other age groups $(\mathrm{p}<0.001)$. No differences in proportions of samples and of positivity were observed between the three epidemic seasons studied.

Influenza like illness incidence rates for the study period were higher for the $0-4 \mathrm{y}$ age group $(\mathrm{p}<0,001)$ (Figure 1). The highest rate ratio (RR) between IV+ rates and ILI rates corresponded to $0-4 \mathrm{y}(0.34 ; 95 \% \mathrm{CI}: 0.28-0.41)$, and the lowest $(0.26 ; 95 \%$ CI: $0.23-0.30)$ to $15-64 y$ (Table 1$)$.

When considering two and three weeks before onset of overall epidemic activity of ILI sentinel reported cases related to overall sentinel population ILI IR during peak activity weeks, the only RR above 1 corresponded to $0-4 \mathrm{y}$ $(1.38 ; 95 \% \mathrm{CI}: 1.08-1.75 ; \mathrm{p}=0.018$ and 1,$29 ; 95 \% \mathrm{CI}: 1.04-1.6$; $\mathrm{p}=0.02$ ) respectively.

The lowest corresponded to $+64 \mathrm{y}(0.06 ; 95 \% \mathrm{CI}: 0.02-0.17$ and $0.09 ; 95 \% \mathrm{CI}: 0.04-0.18$ ) respectively (Table 2 ).

The same occurs for the rate ratio of sentinel IV+ incidence rates related to overall population IR during peak activity weeks where the only RR above 1 corresponded to $0-4$ y $(2.61 ; 95 \% \mathrm{CI}: 1.83,3.72 ; \mathrm{p}<0.001)$ and $(1,95$; $95 \%$ CI:1.39,2.72; $\mathrm{p}=0.001$ ) respectively. (Table 3 )

Table 1. Rate ratio between Influenza virus positive ( IV+) and ILI reported incidence rates during three epidemic seasons studied, by age group and overall population

$\begin{array}{ccc}\text { Season } & \begin{array}{c}\text { Rate ratio IV+/ILI } \\ \text { Age group0-4 }\end{array} & \begin{array}{c}\text { Rate ratio IV+/ILI } \\ \text { Age group 5-14 }\end{array} \\ 2006-07 & 0,45(0,33-0,61) & 0,28(0,20-0,38) \\ 2007-08 & 0,30(0,21-0,43) & 0,27(0,20-0,38) \\ 2008-09 & 0,30(0,22-0,39) & 0,29(0,23-0,37) \\ \text { Overall } & 0,34(0,28-0,41)^{*} & 0,28(0,24-0,33) \\ \text { *p }<\mathbf{0 . 0 0 0 1} & & \end{array}$

Rate ratio IV+/ILI
Age group 15-64
$0,22(0,17-0,29)$
$0,28(0,21-0,37)$
$0,29(0,23-0,36)$
$0,26(0,23-0,30)$
Rate ratio IV+/ILI
Overall
$0,29(0,24-0,34)$
$0,28(0,23-0,33)$
$0,30(0,26-0,34)$
$0,29(0,26-0,32)$


Table 2. Ratio between incidence rates of ILI during 2 (A) and 3(B) weeks prior to onset of epidemic activity by age groups and overall ILI incidence rates reported during the peak epidemic weeks . Influenza surveillance sentinel network of Catalonia (PIDIRAC)

\begin{tabular}{|c|c|c|c|c|c|}
\hline $\mathbf{A}$ & Age group 0-4 & Age group 5-14 & Age group 15-64 & Age group >64 & Overall \\
\hline Season & \multicolumn{5}{|c|}{ Ratio IR-ILI 2 week-prior/overall IR -ILI in peak activity weeks } \\
\hline 2006-07 & $0.67(0.35-1.31)$ & $0.66(0.41-1.06)$ & $0.20(0.14-0.31)$ & $0.04(0.01-0.29)$ & $0.28(0.21-0.38)$ \\
\hline 2007-08 & $1.47(0.97-2.24)$ & $0.57(0.35-0.94)$ & $0.42(0.30-0.60)$ & 0.00 & $0.52(0.40-0.66)$ \\
\hline 2008-09 & $1.74(1.25-2.43)$ & $1.02(0.73-1.42)$ & $0.43(0.31-0.60)$ & $0.15(0.05-0.46)$ & $0.69(0.56-0.86)$ \\
\hline Overall & $\begin{array}{c}1.38(1.08-1.75) * \\
* p=0.018\end{array}$ & $0.77(0.61-0.98)$ & $0.34(0.28-0.42)$ & $0.06(0.02-0.17)$ & $0.49(0.43-0.57)$ \\
\hline B & \multicolumn{5}{|c|}{ Ratio IR-ILI 3 week-prior/overall IR -ILI in peak activity weeks } \\
\hline 2006-07 & $0.81(0.48-1.35)$ & $0.53(0.350 .82)$ & $0.29(0.22-0.39)$ & $0.03(0.01-0.20)$ & $0.32(0.26-0.41)$ \\
\hline 2007-08 & $1.26(0.86-1.85)$ & $0.84(0.59-1.20)$ & $0.35(0.25-0.48)$ & $0.04(0.01-0.28)$ & $0.49(0.39-0.62)$ \\
\hline 2008-09 & $1.64(1.22-2.21)$ & $0.96(0.72-1.29)$ & $0.570 .45-0.73)$ & $0.24(0.11-0.50)$ & $0.75(0.62-0.90)$ \\
\hline Overall & $\begin{array}{c}1.30(1.05-1.60)^{* *} \\
* * \mathrm{p}=0.020\end{array}$ & $0.79(0.65-0.96)$ & $0.39(0.33-0.46)$ & $0.09(0.04-0.18)$ & $0.52(0.46-0.58)$ \\
\hline
\end{tabular}

Table 3. Ratio between incidence rates of IV+ samples during the 2(A) and 3 (B) weeks prior to the onset of epidemic activity by age groups and the overall population IV + sample incidence rates during the peak epidemic weeks. Influenza surveillance sentinel network of Catalonia (PIDIRAC)

\begin{tabular}{|c|c|c|c|c|c|}
\hline $\mathbf{A}$ & Age group 0-4 & Age group 5-14 & Age group 15-64 & Age group $>64$ & Overall \\
\hline Season & \multicolumn{5}{|c|}{ Ratio IR-IV + 2 week-prior/overall IR -IV + in peak activity weeks } \\
\hline 2006-07 & $5.15(3.02-8.42)$ & $1.20(0.64-2.28)$ & $0.60(0.37-0.97)$ & $0.14(0.02-1.08)$ & $0.97(0.68-1.37)$ \\
\hline 2007-08 & $1.30(0.43-3.31)$ & $0.61(0.22-1.68)$ & $0.32(0.15-0.71)$ & $0.23(0.01-1.33)$ & $0.44(0.25-0.76)$ \\
\hline 2008-09 & $1.40(0.67-2.84)$ & $0.99(0.51-1.90)$ & $0.45(0.24-0.83)$ & $0.36(0.09-1.46)$ & $0.67(0.44-1.01)$ \\
\hline Overall & $\begin{array}{c}2.61(1.83-3.72) \\
* * p<0.001\end{array}$ & $1.56(1.12-2.19)$ & $0.60(0.44-0.81)$ & $0.18(0.03-0.57)$ & $0.73(0.58-0.92)$ \\
\hline B & \multicolumn{5}{|c|}{ Ratio IR-IV + 3 week-prior/overall IR -IV + in peak activity weeks } \\
\hline $\begin{array}{l}2006-07 \\
2007-08\end{array}$ & $\begin{array}{l}4.01(2.50-6.39) \\
1.14(0.53-2.48)\end{array}$ & $\begin{array}{l}0.88(0.47-1.65) \\
0.45(0.18-1.12)\end{array}$ & $\begin{array}{c}0.52(0.33-0.80) \\
0.34(0.18-0.63)\end{array}$ & $\begin{array}{l}0.10(0.01-0.74) \\
0.14(0.02-1.02)\end{array}$ & $\begin{array}{c}0.75(0.54-1.05) \\
0.40(0.25-0.64)\end{array}$ \\
\hline 2008-09 & $1.23(0.65-2.29)$ & $1.04(0.61-1.78)$ & $0.59(0.38-0.93)$ & $0.36(0.11-1.13$ & $0.74(0.53-1.04)$ \\
\hline Overall & $\begin{array}{c}1.95(1.39-2.72) * * \\
* * \mathbf{p}=0.001\end{array}$ & $0.82(0.57-1.19)$ & $0.49(0.37-0.65)$ & $0.12(0.04-0.37)$ & $0.65(0.53-0.80)$ \\
\hline
\end{tabular}

Mean incidence ILI rates from 2-3 weeks before onset of peak epidemic activity, mean threshold for overall population set at ILI incidence rates above 90/100,000 persons are significantly higher in the $0-4 \mathrm{y}$ age group $(163.5 / 100,000$ persons) than in the other age groups $(p=0.001)$. Figure 1

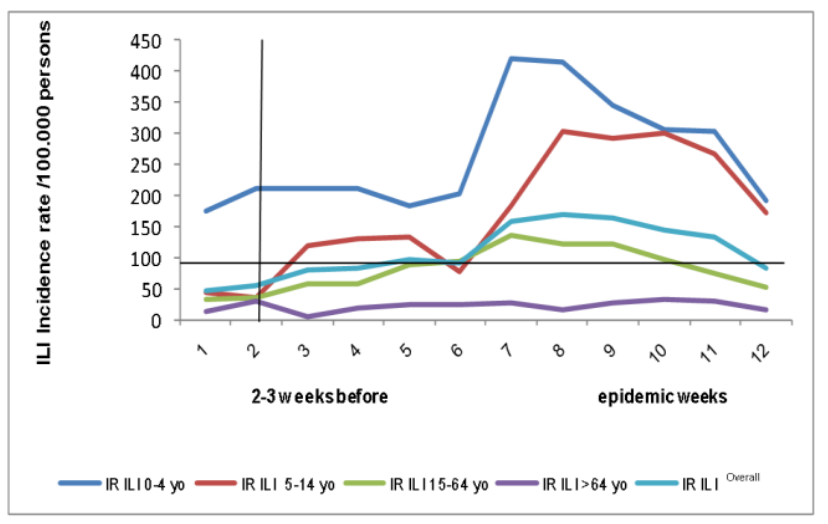

Figure 1. Mean ILI incidence rate for 3 epidemic influenza seasons, by age group and overall ILI incidence rate for the 2-3 weeks period before onset of epidemic activity. Sentinel influenza surveillance network of Catalonia (PIDIRAC)

\section{Discussion}

Sentinel surveillance networks have proven to be an effective tool for detection of influenza activity, yet improvement of this tool could be fostered by determining target indicator groups. Other activity detection methods have been set forth, such as monitoring health seeking behavior in the form of queries to online search engines, which are submitted daily by my millions of users around the world[10].

The fact that overall positivity rate for influenza virus (A and B) was significantly higher in the 5-14 y. than in the other age groups $(p<0.001)$ has also been observed by other authors[11]. Furthermore, several studies have also stated the fact school and pre-school children have an indirect role in anticipating the risk to others and reducing cumulative number of cases[12,13].

Our data show that the information provided by the influenza sentinel surveillance network focused on different age group distribution of consultations and sampling can give valuable information to predict onset of peak epidemic activity in the community. Thus ILI reported and laboratory confirmed incidence has proven to be a suitable indicator to predict onset of influenza peak epidemic activity only when pediatric population is included in the surveillance system; hence the system fails to detect the beginning of the epidemic when only adult population is considered. This fact highlights the relevance of including pediatricians into influenza sentinel surveillance networks. The use of age specific thresholds for influenza incidence rates can be useful in the timeliness prediction and has a high early warning potential[14]. Despite the availability of influenza vaccine, influenza and the diseases associated with it continue to be an important public health issue. Estimating the number of influenza associated cases better reflects the burden of the 
disease in the population and a means for so doing is through the data from sentinel surveillance in the community setting. The impact of influenza activity on hospital admissions in Spain with an overall vaccine coverage in 2003 of $22 \%$ shows an excess hospital admissions not only in the $>64 \mathrm{y}$., with an average national vaccine coverage of $57-63 \%$ in 2003[15] but also in those under 5 y., encouraging the continued use and the strengthening of campaigns to increase vaccination coverage in these age groups because children are a target group to be considered for vaccination in order to diminish household and school transmission of influenza virus in the epidemic influenza season.

Sentinel influenza incidence rates of laboratory confirmed samples distributed according to age groups highlights the timeliness of pediatric to predict the onset of peak seasonal epidemic influenza activity and it could also allow for adequate preparedness to deal with the eventual emergence of an unusual epidemic or pandemic virus.

Furthermore, setting priority for vaccination by targeting the most susceptible (infants and elderly) $[16,17]$ is key in the prevention of high morbidity and mortality due to influenza. Although there is no good evidence that influenza vaccine reduces mortality due to influenza in the elderly[18], the very young and the very old might be better protected by also vaccinating those who are more likely to be in contact with them thereby reducing the risk of exposure, infection and presentation of severe complications due to influenza[19,20]

\section{Conclusions}

In conclusion, it has been stated that immunization of certain age groups may also extend indirect protective benefit to vulnerable populations[12]. Identifying which age group(s) are related to earliest seasonal influenza activity and making them eligible for vaccination enhances their indirect impact at community level. In conclusion, our results show a high correspondence between ILI and IV+ IR observed in children 0-4y; in this age group rates of ILI during two and three consecutive weeks prior to increase above threshold activity can be used to predict the onset of peak epidemic activity in a timely manner.

\section{ACKNOWLEDGEMENTS}

This work was partially supported by CIBER Epidemiología y Salud Pública (CIBERESP), Spain and Agència de Gestió d'Ajuts Universitaris i de Recerca AGAUR[ GR09/3 30].

The other members of the PIDIRAC sentinel surveillance network of Catalonia during the influenza epidemic seasons studied: Marcos, $\mathrm{M}^{\mathrm{a}}$ Angeles; de Molina, Patricia (Microbiology Service, H Clinic of Barcelona). Laura Ruiz, Basile Luca (Directorate of Public Health. Department of Health. Generalitat of Catalonia), Aizpurua Galdeano, Ma. Pilar; Alonso Ciruelos, Joaquim ;Arigon Gaudiano, Pablo; Azemar Mallard, Juan; Basas Prat, Dolors; Benaiges Mon- real,
Domènec; Besora Anglerill, Roser; Bravo Corrales, Jose Luís; Callado Coma Montserrat ;Casanovas Gordo, Jose $\mathrm{M}^{\mathrm{a}}$; Cid Xutgla, Ada; Ciurana Roca, Emili; Cots Yago, Jose M; de la Rica Escuin, David; Domingo Fuster, Maria ; Elizalde del Rio, Guillermo; Estabanell Buxo, Ana; Fau Montllor, Eugeni; Fernandez Fernandez, Olga; Forcada Arcarons, Anna; Forga Visa, F Xavier; Fos Escrivà, Emili ;Gadea Borguño, Gloria; Jordi Garcia Perez, Jordi; Gatius Tonda, Carles; Grivé Isern, Montserrat ; Guzman Bonilla, Maria Cruz; Juscafresa Mir, Dolors; Leon Marin, Irma ;Llusa Parramon, Ana Maria; Lopez Mompo, Cristina; Macia Rieradevall, M Esperança; ; Mainou Alicia ; Marco Segarra, Emili ; Martinez Fernandez, Mercedes; Martinez Jimenez, Eugenia; Molinero Egea Cesar; Moncosí de Borbon Xavier ; Naranjo Orijuela, Angeles; Navarro Martin, David; Ortolà Castells, M ${ }^{\mathrm{a}}$ Eugenia; Perez Cifuentes, M Mar; Perez Centellas Carmen ;Pozo Diaz, Cristina; Prat Figueras, Manel; Pujol Salud, Jesús; Pujol Martinez, Ramon; Ribatallada Diez, Anna Maria; Ruiz Aragón, Gloria; Sanchez Pellicer, Ramon; Sarra Manetas, Nuria; Valen Suñer, Elena; Valencia Pedraza, Ivan; van Esso Arbolave, Diego; Vila Pablos Carme; Zabala Charramendieta, Eduardo; Zurilla Leonarte, Elena.

\section{REFERENCES}

[1] Weil-Olivier C.[Seasonal influenza in children]. Rev Prat 2008 Oct 15;58(15):1661-6.

[2] Peltola V, Ziegler T, Ruuskanen O. Influenza A and B virus infections in children. Clin Infect Dis 2003 Feb $1 ; 36(3): 299-305$.

[3] Heikkinen T, Ziegler T, Peltola V, Lehtinen P, Toikka P, Lintu M, et al. Incidence of influenza in Finnish children. Pediatr Infect Dis J 2003 Oct;22(10 Suppl):S204-S206.

[4] Ortiz JR, Sotomayor V, Uez OC, Oliva O, Bettels D, McCarron M, et al. Strategy to enhance influenza surveillance worldwide. Emerg Infect Dis 2009 Aug;15(8): 1271-8.

[5] Jefferson T, Smith S, Demicheli V, Harn den A, Rivetti A, Pietrantonj C Di. Assessment of the effi- cacy and effectiveness of influenza vaccines in healthy children:systematic review. Lancet 2005,365:773-780.

[6] Jefferson T, Rivetti D, Rivetti A, Rudin M, Pietra- tonj C Di, Demicheli V. Efficacy and effectiveness of influenza vaccines in elderly people: a systematic review. Lancet 2005 , 366:1165-1174.

[7] Reichert TA, Sugaya N, Fedson DS, Glezen WP, Simonsen L, Tashiro T. The Japanese experience with vaccinating schoolchildren against influenza. N Engl J Med 2001, 44:889-896.

[8] Glezen WP. Emerging infections: pandemic influenza. Epidemiol Rev 1996,18:64-76.

[9] European Centre for Disease Prevention and Control. Protocol for case-control studies to measure influenza vaccine effectiveness in the European Union and European Economic Area Member States. Available Onlinehttp://ecdc.europa.eu/ 
en/publications/Publications/0907_TED_Influenza_AH1N1 Measur ing_Influenza_Vaccine_Effectiveness_Protocol_Ca e_Control_Studies.pdf

[10] Ginsberg J, Mohebbi MH, Patel RS, Brammer L, Smolinski MS, Brilliant L. Detecting influenza epidemics using search engine query data. Nature 2009 Feb 19; 457(7232):1012-4.

[11] Reina J, Nicolau A, Galmes A, Arbona B.[Diagnostic yield of pediatric respiratory samples in the Balearic Islands Sentinel Influenza Surveillance Network.]. An Pediatr (Barc) 2009 Apr 16; 70(5):438-42.

[12] Sebastian R, Skowronski DM, Chong M, Dhaliwal J, Brownstein JS. Age-related trends in the timeliness and prediction of medical visits, hospitalizations and deaths due to pneumonia and influenza, British Columbia, Canada, 1998-2004. Vaccine 2008 Mar 4;26(10):1397-403.

[13] Cauchemez S, Valleron AJ, Boelle PY, Flahault A, Ferguson NM. Estimating the impact of school closure on influenza transmission from Sentinel data. Nature 2008 Apr 10; 452(7188):750-4.

[14] Cooper DL, Verlander NQ, Elliot AJ, Joseph CA, Smith GE. Can syndromic thresholds provide early warning of national influenza outbreaks? J Public Health (Oxf) 2009 Mar;31(1):17-25.
[15] Vazquez-Fernandez DP, Hernandez-Barrera V, Carasco-Garrido P, Gil M, Jimenez-Garcia R. [Changes in influenza vaccination coverage in Spain from 2001 to 2003. Analysis by autonomous communities. Gaceta Sanitaria 2007;21:10-7.

[16] Stevenson E, Barrios L, Cordell R, de Lozier D, Gorman S, Koenig LJ, et al. Pandemic influenza planning: addressing the needs of children. Am J Public Health 2009 Oct;99 Suppl 2:S255-S260.

[17] Hutchins SS, Truman BI, Merlin TL, Redd SC. Protecting vulnerable populations from pandemic influenza in the United States: a strategic imperative. Am J Public Health 2009 Oct; 99 Suppl 2:S243-S248.

[18] Jefferson T, Di PC, Al-Ansary LA, Ferroni E, Thorning S, Thomas RE. Vaccines for preventing influenza in the elderly. Cochrane Database Syst Rev 2010; (2):CD004876.

[19] Thomas RE, Jefferson T, Lasserson TJ. Influenza vaccination for healthcare workers who work with the elderly: systematic review. Vaccine 2010 Dec 16;29(2):344-56.

[20] Ying-Hen Hshieh. Age grups and spread of influenza:implications for vaccination strategy. BMC Infectious Diseases 2010[cited 2011 Apr 15]; Available Online: http://www.biomedcentral.com/1471-2334/10/106 